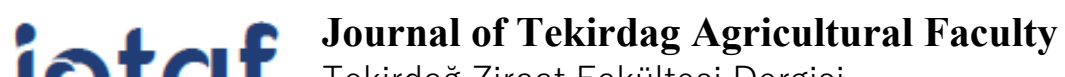 \\ Tekirdağ Ziraat Fakültesi Dergisi
}

\section{Germination and Early Growth Performances of Mung Bean (Vigna radiata (L.) Wilczek) Genotypes Under Salinity Stress}

\author{
Tuzluluk Stresi Altındaki Maş Fasulyesi (Vigna radiata (L.) Wilczek) Genotiplerinin \\ Çimlenme ve Erken Gelişme Performansları
}

\section{Berk BENLIOGLU ${ }^{1 *}$, Ugur OZKAN ${ }^{1}$}

\begin{abstract}
Salinity is the abiotic stress factor that most restricts agricultural production after drought. In this study, mung beans (Vigna radiata (L.) Wilczek) genotypes were analyzed the resistance performance to salinity stress in germination stage and early seedling stage. In accordance with this purpose, 17 mung bean genotypes were used in the study. Genotypes used in the study were treated with distilled water ( 0$)$ and 2 different salt doses (4 and 8 mmhos $\left.\mathrm{cm}^{-1} \mathrm{NaCl}\right)$ as the control group. Petri dishes were allowed to stay at room temperature $\left(25^{\circ} \mathrm{C}\right)$ for 9 days, and salt solution was added when required. The data obtained from the study, were obtained on the average of the measurements made on the $9^{\text {th }}$ day and then analyzed. Germination power, root length, shoot length, fresh weight and dry weight were determined as the analyzed parameters in the study. Increasing $\mathrm{NaCl}$ doses generally affected all parameters negatively. According to the analysis of variance with the results obtained, the genotypic effect was found to be statistically significant in all parameters, which was analyzed in this study. Stress dose was found to be statistically significant in all parameters except the dry weight parameter. In addition to this, genotype $\times$ stress dose interaction was determined to be statistically significant in germination power, root length and shoot length parameters. Genotypes, which were numbered No.20 and No.24, had maximum results in statistically important parameters such as germination power (97.00-94.11\%), root length $(1.557-1.563 \mathrm{~cm})$ and shoot length $(2.033$ $1.793 \mathrm{~cm}$ ) under applied highest salt dose. As a result of this, No.20 and No.24 genotypes were determined to be the more tolerant to salt stress than other genotypes used in the study. As a conclusion, it is suggested that No.20 and No.24 genotypes can be used as parental plants in the breeding of new varieties tolerant to salinity in the future.
\end{abstract}

Keywords: Vigna radiata (L.) Wilczek, Abiotic stress, $\mathrm{NaCl}$, Germination rate, Early seedling

Özet

Tuzluluk kuraklıktan sonra tarımsal üretimi en fazla sınırlayan abiyotik stres faktörüdür. Bu çalışmada bazı maş fasulyesi (Vigna radiata (L.) Wilczek) genotiplerinin çimlenme dönemi ve erken gelişme döneminde tuza karşı olan dayanıklılık performansları incelenmiştir. Bu amaç doğrultusunda çalışmada 17 maş fasulyesi genotipi kullanılmıştır. Çalışmada kullanılan genotipler, kontrol grubu olarak saf su (0) ve 2 farklı tuz dozu (4 ve 8 mmhos $\left.\mathrm{cm}^{-1} \mathrm{NaCl}\right)$ ile muamele edilmiştir. Petri kaplarının 9 gün boyunca oda sıcaklığında $\left(25^{\circ} \mathrm{C}\right)$ beklemesi sağlanmıştır ve gereken durumlarda tuz çözeltisi ilave edilmiştir. Çalışmadan elde edilen veriler 9. günde yapılan ölçümlerin ortalaması üzerinden elde edilerek analize tabi tutulmuştur. Çimlenme gücü, kök uzunluğu, sürgün uzunluğu, yaş ağırlık ve kuru ağırlık çalışmada incelenen parametreler olarak belirlenmiştir. NaCl dozunda görülen artış incelenen tüm parametreleri olumsuz bir şekilde etkilenmiştir. Elde edilen sonuçlara göre yapılan varyans

\footnotetext{
1*Sorumlu Yazar/Corresponding Author: Berk BENLIOGLU, Ankara University, Faculty of Agriculture, Field Crops Department, Diskapi, ANKARA. E-mail: benliogluberk@hotmail.com (iD) OrcID: 0000-0002-2400-057X.

${ }^{2}$ Ugur OZKAN, Ankara University, Faculty of Agriculture, Field Crops Department, Diskapi. E-mail: ugurozkan@ankara.edu.tr (D) OrcID: 0000-0002-68694526 .

Atıf/Citation: Benlioglu, B., Ozkan, U. Germination and Early Growth Performances of Mung Bean (Vigna radiata (L.) Wilczek) Genotypes Under Salinity Stress. Tekirdă̆ Ziraat Fakültesi Dergisi, 17(3), 318-328.

CBu çalışma Tekirdağ Namık Kemal Üniversitesi tarafından Creative Commons Lisansı (https://creativecommons.org/licenses/by-nc/4.0/) kapsamında yayınlanmıştır. Tekirdağ 2020 
analizinde genotipik etki tüm çalışmada incelenen parametrelerde istatistiksel olarak önemli bulunmuştur. Stres dozu kuru ağırlık parametresi dışında kalan tüm parametrelerde istatistiksel olarak önemli olduğu bulunmuştur. Buna ek olarak, genotip $\times$ stres dozu interaksiyonunun ise çimlenme gücü, kök uzunluğu ve sürgün uzunluğu parametrelerinde istatistiksel olarak önemli olduğu belirlenmiştir. No.20 ve No.24 olarak numaralandırılan genotipler, uygulanan en yüksek tuz dozu altında çimlenme gücü $(97.00-94.11 \%)$, kök uzunluğu $(1.557-1.563 \mathrm{~cm})$ ve sürgün uzunluğu (2.033-1.793 cm) gibi istatistiksel olarak önemli parametrelerde, tüm genotipler içerisinde en yüksek değerleri elde etmişlerdir. Bunun sonucu olarak, No.20 ve No.24 numaralı genotipler, çalışmada kullanılan diğer genotiplerden tuz stresine karşı daha toleranslı oldukları belirlenmiştir. Sonuç olarak, No.20 ve No.24 numaralı genotiplerin gelecekte tuzluluğa dayanıklı yeni çeşitlerin yetiştirilmesinde ebeveyn bitki olarak kullanılabileceği önerilmektedir.

Anahtar Kelimeler: Vigna radiata (L.) Wilczek, Abiyotik stres, $\mathrm{NaCl}$, Çimlenme oranı, Erken fide 


\section{Introduction}

Mung bean (Vigna radiata (L.) Wilczek) is a genus with high nutritional content and high digestibility in the food legumes (Siemonsma and Na Lampang, 1992). However, mung bean, which has a warm climate, has not been sufficiently widespread in the world due to the lack of recognition and the cultivation of approximately 6 million hectare (HanumanthaRao et al., 2016). Mung bean vegetation period is short (65-90 days), has a wide adaptability, relatively less need for water compared to other legume plants (Parida and Das, 2005) and low input costs in production, high protein value (22-28\%) and nutritional value (Singh and Singh, 2011). In particular, where monoculture agriculture is widespread and access to protein resources in nutrition is limited, it will benefit both ecologically for producers and consumers.

Agricultural areas in the world have reached their natural limits. There are losses in agricultural areas due to global warming, distorted urbanization and some industrial activities. In addition, these areas become inefficient due to erosion, wasteland and faulty agricultural activities. The only measure that can be taken to meet the nutritional needs of the growing population is to increase the amount of product obtained from the unit area $(\mathrm{kg}$ $\mathrm{da}^{-1}$ or ton $\left.\mathrm{ha}^{-1}\right)$. Within this, it is necessary to develop new varieties resistant to biotic and abiotic stress factors and to determine the tolerance levels of plants, species and varieties to these stress factors (Ozgen et al., 2005).

Salinity is the abiotic stress factor that most restricts agricultural production after drought. Today, about $20 \%$ of the world's crop production areas and about $50 \%$ of irrigated agricultural areas are affected by salinity (Zhu, 2001; Pitman and Läuchli, 2002; Tuteja, 2007). There are about 1.6 million hectares land, which has salinity and alkalinity problem, in Turkey (Demirbas and Alkan, 2018). has been found to have a problem. Salinity stress, which occurs in the form of osmotic stress and ion stress in plants, prevents growth and development; structural, physiological, biochemical and molecular level changes (Culha and Cakirlar, 2011). Salinity stress causes a decrease in yield in mung beans (Abd-Alla et al., 1998; Saha et al., 2010), seed germination, root and shoot lengths, age and dry seedling weights decrease and this varies significantly depending on genotypes (Promila and Kumar, 2000; Misra and Dwivedi, 2004). Mung bean has been reported to tolerate 5-6 mmhos $\mathrm{cm}^{-1}$ salinity during germination period (Singh and Singh, 2011).

In this study, genotypes were selected as high yield property of mung beans, the most abundant form of salt in soil and the levels of tolerance to salt stress in germination and early seedling stage by using the identify have been made.

\section{Materials and Methods}

17 mung bean genotypes were used in the study. The locations of genotypes are presented in table 1.

Seeds were germinated between filter papers placed in petri dishes in accordance with ISTA rules (ISTA, 2016). Genotypes were treated with pure water $(0)$ as the control group and 2 different doses $\left(4\right.$ and $\left.8 \mathrm{mmhos} \mathrm{cm}^{-1}\right)$ of $\mathrm{NaCl}$, the most common salt form in soils. Petri dishes were allowed to stand at room temperature $\left(25 \mathrm{C}^{\circ}\right)$ for 9 days and salt solution was added when necessary. Data were obtained by measurements and counts on day 9 .

"Germination power", "root length", "shoot length", fresh weight" and "dry weight" were determined by randomly choosing 15 seeds of each from petri dishes. Also, they were measured, counted and averaged their numbers. In order to calculate the dry weight, fresh shoots were dried for 72 hour at $60 \mathrm{C}^{\circ}$ (Ylldı and Ozgen, 2004).

The data obtained in the study were subjected to analysis of variance in accordance with the completely randomized design with three replication using JMP-12 statistics package program (SAS, 2015) to compare significant differences among treatments. Duncan's multiple range test was applied to compare the means if there were any significant differences. 
Table 1. Locations of genotypes

\begin{tabular}{cccc}
\hline $\begin{array}{c}\text { Genotype } \\
\text { Number }\end{array}$ & Location & $\begin{array}{c}\text { Genotype } \\
\text { Number }\end{array}$ & Location \\
\hline 1 & Adiyaman-Turkey & 27 & Gaziantep-Turkey \\
2 & Adiyaman-Turkey & 44 & Mersin-Turkey \\
3 & Adiyaman-Turkey & 79 & Karaman-Turkey \\
5 & Adiyaman-Turkey & 81 & Karaman-Turkey \\
12 & Antalya-Turkey & 91 & Kilis-Turkey \\
15 & Antalya-Turkey & 96 & Uzbekistan \\
20 & Antalya-Turkey & 98 & Uzbekistan \\
21 & Antalya-Turkey & 99 & Turkmenistan \\
24 & Gaziantep-Turkey & & \\
\hline
\end{tabular}

\section{Results and Discussion}

The mean values of the parameters examined and Duncan groups are presented in Table 2.

\subsection{Germination power}

Genotype (dF:16; Ms: 262.589), $\mathrm{NaCl}(\mathrm{dF}: 2 ; \mathrm{Ms}: 3,790.625)$ and Genotype $\times \mathrm{NaCl}$ interaction (dF:32; Ms: 82.795) had statistically different $(p<0.01)$. It is seen that the germination power decreases with increasing salinity stress (Table 2, Figure 1). The mean germination power of genotypes ranges from 97.00 to $85.77 \%$. With the onset of salt stress, germination of genotype "No.12" occurred dramatically, and the highest stress dose was the least germinated genotype at 8 mmos $\mathrm{cm}^{-1}$. Genotypes "No.20" and "No.24" genotype have the highest germination power $(91.00 \%)$ while maintaining their germination potential at $8 \mathrm{mmhos} \mathrm{cm}^{-1}$ salt dose (Table 2).

Salinity stress is particularly effective in germination mung bean (Paliwal and Maliwal, 1980). While genotypes generally maintained germination characteristics of $5 \mathrm{mmhos} \mathrm{cm}^{-1}$, germination decreased after this stress level. These results are in agreement with previous studies on mung beans (Promila and Kumar, 2000; Misra and Dwivedi, 2004; Saha et al., 2010).

Table 2. The mean values of mung bean genotypes of germination parameters under different salt stress level

\begin{tabular}{cllll} 
& \multicolumn{3}{c}{ Germination Power (\%) } & \\
\cline { 2 - 4 } Genotypes & Control (0) & $\mathbf{4} \mathbf{~ m m h o s ~ c m} \mathbf{~}^{-1}$ & $\mathbf{8 ~ m m h o s ~ \mathbf { ~ m } ^ { - 1 }}$ & \multicolumn{1}{c}{ Mean } \\
\hline 1 & $97.67 \mathrm{a}-\mathrm{c}$ & $88.67 \mathrm{~b}-\mathrm{g}$ & $77.33 \mathrm{~h}$ & $87.89 \mathrm{CD}$ \\
2 & $95.33 \mathrm{a}-\mathrm{d}$ & $90.67 \mathrm{a}-\mathrm{f}$ & $82.00 \mathrm{f}-\mathrm{h}$ & $89.33 \mathrm{~B}-\mathrm{D}$ \\
3 & $95.33 \mathrm{a}-\mathrm{d}$ & $93.00 \mathrm{a}-\mathrm{e}$ & $84.33 \mathrm{e}-\mathrm{h}$ & $90.89 \mathrm{~B}-\mathrm{D}$ \\
5 & $100.00 \mathrm{a}$ & $97.67 \mathrm{a}-\mathrm{c}$ & $81.67 \mathrm{f}-\mathrm{h}$ & $93.11 \mathrm{~A}-\mathrm{C}$ \\
12 & $95.20 \mathrm{a}-\mathrm{d}$ & $66.63 \mathrm{i}$ & $55.53 \mathrm{j}$ & $72.46 \mathrm{E}$ \\
15 & $97.67 \mathrm{a}-\mathrm{c}$ & $88.67 \mathrm{~b}-\mathrm{g}$ & $78.67 \mathrm{~h}$ & $88.33 \mathrm{CD}$ \\
20 & $100.00 \mathrm{a}$ & $100.00 \mathrm{a}$ & $91.00 \mathrm{a}-\mathrm{f}$ & $97.00 \mathrm{~A}$ \\
21 & $97.67 \mathrm{a}-\mathrm{c}$ & $93.00 \mathrm{a}-\mathrm{e}$ & $79.67 \mathrm{~g}-\mathrm{h}$ & $90.11 \mathrm{~B}-\mathrm{D}$ \\
24 & $96.00 \mathrm{a}-\mathrm{c}^{1}$ & $95.33 \mathrm{a}-\mathrm{d}$ & $91.00 \mathrm{a}-\mathrm{f}$ & $94.11 \mathrm{AB}$ \\
27 & $97.67 \mathrm{a}-\mathrm{c}$ & $95.33 \mathrm{a}-\mathrm{d}$ & $88.33 \mathrm{c}-\mathrm{g}$ & $93.78 \mathrm{AB}$ \\
44 & $97.67 \mathrm{a}-\mathrm{c}$ & $91.00 \mathrm{a}-\mathrm{f}$ & $86.33 \mathrm{~d}-\mathrm{h}$ & $91.67 \mathrm{~A}-\mathrm{C}$ \\
79 & $100.00 \mathrm{a}$ & $95.33 \mathrm{a}-\mathrm{d}$ & $88.33 \mathrm{c}-\mathrm{g}$ & $94.56 \mathrm{AB}$ \\
81 & $97.67 \mathrm{a}-\mathrm{c}$ & $100.00 \mathrm{a}$ & $77.33 \mathrm{~h}$ & $91.67 \mathrm{~A}-\mathrm{C}$ \\
91 & $100.00 \mathrm{a}$ & $95.33 \mathrm{a}-\mathrm{d}$ & $84.00 \mathrm{e}-\mathrm{h}$ & $93.11 \mathrm{~A}-\mathrm{C}$ \\
96 & $97.77 \mathrm{a}-\mathrm{b}$ & $95.53 \mathrm{a}-\mathrm{d}$ & $64.43 \mathrm{ij}$ & $85.91 \mathrm{D}$ \\
98 & $97.67 \mathrm{a}-\mathrm{c}$ & $97.67 \mathrm{a}-\mathrm{c}$ & $84.33 \mathrm{e}-\mathrm{h}$ & $93.22 \mathrm{~A}-\mathrm{C}$ \\
99 & $95.53 \mathrm{a}-\mathrm{d}$ & $93.20 \mathrm{a}-\mathrm{e}$ & $79.87 \mathrm{~g}-\mathrm{h}$ & $89.53 \mathrm{~B}-\mathrm{D}$ \\
Mean & $97.58 \mathrm{~A}$ & $92.77 \mathrm{~B}$ & $80.83 \mathrm{C}$ & 90.39 \\
\hline
\end{tabular}


Table 2. continued

\begin{tabular}{|c|c|c|c|c|}
\hline \multirow[b]{2}{*}{ Genotypes } & \multicolumn{3}{|c|}{ Root Length (cm) } & \multirow[b]{2}{*}{ Mean } \\
\hline & Control (0) & $4 \mathrm{mmhos} \mathrm{cm}^{-1}$ & $8 \mathrm{mmhos} \mathrm{cm}^{-1}$ & \\
\hline 1 & $6.287 \mathrm{a}-\mathrm{c}$ & $3.370 \mathrm{~d}-\mathrm{k}$ & 1.260 i-ö & $3.639 \mathrm{AB}$ \\
\hline 2 & $6.233 \mathrm{a}-\mathrm{c}$ & $3.113 \mathrm{~d}-\mathrm{m}$ & $0.743 \mathrm{k}-\mathrm{m}$ & $3.363 \mathrm{BC}$ \\
\hline 3 & 5.410 a-e & $3.700 \mathrm{c}-\mathrm{j}$ & $1.293 \mathrm{i}-\mathrm{m}$ & $3.468 \mathrm{~A}-\mathrm{C}$ \\
\hline 5 & $6.553 \mathrm{ab}$ & $3.770 \mathrm{~b}-\mathrm{i}$ & $0.937 \mathrm{j}-\mathrm{m}$ & $3.753 \mathrm{AB}$ \\
\hline 12 & $1.767 \mathrm{~h}-\mathrm{m}$ & $0.873 \mathrm{k}-\mathrm{m}$ & $0.740 \mathrm{k}-\mathrm{m}$ & $1.127 \mathrm{G}$ \\
\hline 15 & $3.767 \mathrm{c}-\mathrm{i}$ & $2.367 \mathrm{f}-\mathrm{m}$ & $0.867 \mathrm{k}-\mathrm{m}$ & $2.333 \mathrm{DE}$ \\
\hline 20 & $5.783 \mathrm{a}-\mathrm{d}$ & $2.827 \mathrm{e}-\mathrm{m}$ & $1.557 \mathrm{~h}-\mathrm{m}$ & $3.389 \mathrm{BC}$ \\
\hline 21 & $2.090 \mathrm{~g}-\mathrm{m}$ & $0.870 \mathrm{k}-\mathrm{m}$ & $0.577 \mathrm{~m}$ & $1.179 \mathrm{G}$ \\
\hline 24 & $5.660 \mathrm{a}-\mathrm{d}$ & $2.670 \mathrm{e}-\mathrm{m}$ & $1.563 \mathrm{~h}-\mathrm{m}$ & $3.298 \mathrm{BC}$ \\
\hline 27 & $1.593 \mathrm{~h}-\mathrm{m}$ & $1.150 \mathrm{i}-\mathrm{m}$ & $1.113 \mathrm{i}-\mathrm{m}$ & $1.286 \mathrm{FG}$ \\
\hline 44 & $4.933 \mathrm{~b}-\mathrm{f}$ & $2.007 \mathrm{~h}-\mathrm{m}$ & $0.510 \mathrm{~m}$ & $2.483 \mathrm{D}$ \\
\hline 79 & $2.727 \mathrm{e}-\mathrm{m}$ & $1.427 \mathrm{~h}-\mathrm{m}$ & $0.953 \mathrm{j}-\mathrm{m}$ & $1.702 \mathrm{E}-\mathrm{G}$ \\
\hline 81 & $7.827 \mathrm{a}$ & $3.363 \mathrm{~d}-1$ & $1.300 \mathrm{i}-\mathrm{m}$ & $4.163 \mathrm{~A}$ \\
\hline 91 & $2.160 \mathrm{f}-\mathrm{m}$ & $1.330 \mathrm{i}-\mathrm{m}$ & $0.933 \mathrm{j}-\mathrm{m}$ & $1.474 \mathrm{FG}$ \\
\hline 96 & $4.800 \mathrm{~b}-\mathrm{g}$ & $2.570 \mathrm{f}-\mathrm{m}$ & $1.000 \mathrm{i}-\mathrm{m}$ & $2.790 \mathrm{CD}$ \\
\hline 98 & $4.210 \mathrm{~b}-\mathrm{h}$ & $4.913 \mathrm{~b}-\mathrm{f}$ & $1.143 \mathrm{i}-\mathrm{m}$ & $3.422 \mathrm{~A}-\mathrm{C}$ \\
\hline 99 & $3.210 \mathrm{~d}-\mathrm{m}$ & $2.310 \mathrm{f}-\mathrm{m}$ & $0.5831-\mathrm{m}$ & $2.034 \mathrm{D}-\mathrm{F}$ \\
\hline Mean & $4.412 \mathrm{~A}$ & $2.508 \mathrm{~B}$ & $1.004 \mathrm{C}$ & 2.641 \\
\hline \multicolumn{5}{|c|}{ Shoot Length $(\mathrm{cm})$} \\
\hline 1 & $4.087 \mathrm{a}-\mathrm{e}$ & $1.857 \mathrm{f}-\mathrm{o}$ & $1.643 \mathrm{~h}-\mathrm{o}$ & $2.529 \mathrm{~B}-\mathrm{D}$ \\
\hline 2 & $3.177 \mathrm{c}-\mathrm{h}$ & $1.833 \mathrm{~g}-\mathrm{o}$ & $1.003 \mathrm{~m}-\mathrm{o}$ & $2.004 \mathrm{E}$ \\
\hline 3 & $4.993 \mathrm{a}$ & $2.187 \mathrm{f}-\mathrm{n}$ & $1.100 \mathrm{k}-\mathrm{o}$ & $2.760 \mathrm{~A}-\mathrm{B}$ \\
\hline 5 & $3.233 \mathrm{c}-\mathrm{g}$ & $1.853 \mathrm{f}-\mathrm{o}$ & $0.983 \mathrm{~m}-\mathrm{o}$ & $2.023 \mathrm{E}$ \\
\hline 12 & $1.157 \mathrm{k}-\mathrm{o}$ & $0.917 \mathrm{~m}-\mathrm{o}$ & 0.707 no & $0.927 \mathrm{G}$ \\
\hline 15 & $3.400 \mathrm{~b}-\mathrm{f}$ & $1.867 \mathrm{f}-\mathrm{o}$ & $1.633 \mathrm{~h}-\mathrm{o}$ & $2.300 \mathrm{C}-\mathrm{E}$ \\
\hline 20 & $5.413 \mathrm{a}$ & $1.900 \mathrm{f}-\mathrm{o}$ & $2.033 \mathrm{f}-\mathrm{o}$ & $3.116 \mathrm{~A}$ \\
\hline 21 & $4.853 \mathrm{ab}$ & $2.190 \mathrm{f}-\mathrm{n}$ & $1.323 \mathrm{j}-\mathrm{o}$ & $2.789 \mathrm{AB}$ \\
\hline 24 & $2.350 \mathrm{f}-\mathrm{m}$ & $2.127 \mathrm{f}-\mathrm{o}$ & $1.793 \mathrm{~g}-\mathrm{o}$ & $2.090 \mathrm{E}$ \\
\hline 27 & $4.420 \mathrm{a}-\mathrm{c}$ & $2.653 \mathrm{e}-\mathrm{k}$ & $1.783 \mathrm{~g}-\mathrm{O}$ & $2.952 \mathrm{AB}$ \\
\hline 44 & $2.793 \mathrm{~d}-\mathrm{j}$ & $2.323 \mathrm{f}-\mathrm{m}$ & $1.457 \mathrm{i}-\mathrm{o}$ & $2.191 \mathrm{DE}$ \\
\hline 79 & $4.980 \mathrm{a}^{\circ}$ & $2.567 \mathrm{e}-1$ & $1.567 \mathrm{i}-\mathrm{o}$ & $3.038 \mathrm{~A}$ \\
\hline 81 & $1.723 \mathrm{~g}-\mathrm{o}$ & $1.420 \mathrm{i}-\mathrm{o}$ & 0.760 no & $1.301 \mathrm{FG}$ \\
\hline 91 & $4.287 \mathrm{a}-\mathrm{d}$ & $2.387 \mathrm{f}-\mathrm{m}$ & $1.427 \mathrm{i}-\mathrm{o}$ & $2.700 \mathrm{~A}-\mathrm{C}$ \\
\hline 96 & $1.873 \mathrm{f}-\mathrm{o}$ & $1.187 \mathrm{k}-\mathrm{o}$ & $1.0331-0$ & $1.364 \mathrm{~F}$ \\
\hline 98 & $2.930 \mathrm{c}-\mathrm{i}$ & $2.043 \mathrm{f}-\mathrm{o}$ & $1.640 \mathrm{~h}-\mathrm{o}$ & $2.204 \mathrm{DE}$ \\
\hline 99 & $1.210 \mathrm{k}-\mathrm{o}$ & $1.103 \mathrm{k}-\mathrm{o}$ & $0.573 \mathrm{o}$ & $0.962 \mathrm{FG}$ \\
\hline Mean & $3.346 \mathrm{~A}$ & $1.907 \mathrm{~B}$ & $1.321 \mathrm{C}$ & 2.191 \\
\hline \multicolumn{5}{|c|}{ Fresh Weight (g) } \\
\hline 1 & 1.837 & 1.040 & 0.873 & $1.250 \mathrm{H}-\mathrm{J}$ \\
\hline 2 & 1.765 & 1.105 & 0.768 & $1.213 \mathrm{IJ}$ \\
\hline 3 & 1.819 & 1.358 & 0.682 & $1.286 \mathrm{G}-\mathrm{I}$ \\
\hline 5 & 1.411 & 1.034 & 0.677 & $1.041 \mathrm{~J}$ \\
\hline 12 & 2.321 & 1.866 & 1.552 & $1.913 \mathrm{~B}-\mathrm{D}$ \\
\hline 15 & 2.375 & 1.892 & 1.544 & $1.937 \mathrm{~B}-\mathrm{D}$ \\
\hline 20 & 1.965 & 2.002 & 2.008 & $1.991 \mathrm{BC}$ \\
\hline 21 & 1.698 & 1.323 & 1.164 & $1.395 \mathrm{~F}-\mathrm{I}$ \\
\hline 24 & 1.671 & 1.325 & 1.405 & $1.467 \mathrm{~F}-\mathrm{H}$ \\
\hline 27 & 1.731 & 1.480 & 1.235 & $1.482 \mathrm{FG}$ \\
\hline 44 & 1.866 & 1.846 & 1.539 & $1.750 \mathrm{DE}$ \\
\hline 79 & 1.544 & 1.091 & 1.031 & $1.222 \mathrm{IJ}$ \\
\hline 81 & 2.104 & 1.774 & 1.892 & $1.923 \mathrm{~B}-\mathrm{D}$ \\
\hline 91 & 2.302 & 2.142 & 1.746 & $2.063 \mathrm{~B}$ \\
\hline 96 & 2.829 & 2.503 & 2.201 & $2.511 \mathrm{~A}$ \\
\hline 98 & 1.978 & 1.550 & 1.252 & $1.593 \mathrm{EF}$ \\
\hline 99 & 2.141 & 1.775 & 1.529 & $1.815 \mathrm{C}-\mathrm{E}$ \\
\hline Mean & $1.962 \mathrm{~A}$ & $1.594 \mathrm{~B}$ & $1.359 \mathrm{C}$ & 1.638 \\
\hline
\end{tabular}


Table 2. continued

\begin{tabular}{|c|c|c|c|c|}
\hline \multirow[b]{2}{*}{ Genotypes } & \multicolumn{3}{|c|}{ Dry Weight (g) } & \multirow[b]{2}{*}{ Mean } \\
\hline & Control (0) & $4 \mathrm{mmhos} \mathrm{cm}^{-1}$ & $8 \mathrm{mmhos} \mathrm{cm}^{-1}$ & \\
\hline 1 & 0.215 & 0.223 & 0.247 & $0.228 \mathrm{~F}$ \\
\hline 2 & 0.204 & 0.233 & 0.268 & $0.235 \mathrm{~F}$ \\
\hline 3 & 0.187 & 0.222 & 0.235 & $0.215 \mathrm{~F}$ \\
\hline 5 & 0.191 & 0.210 & 0.241 & $0.214 \mathrm{~F}$ \\
\hline 12 & 0.633 & 0.643 & 0.594 & $0.623 \mathrm{AB}$ \\
\hline 15 & 0.463 & 0.532 & 0.515 & $0.503 \mathrm{~B}-\mathrm{E}$ \\
\hline 20 & 0.344 & 0.359 & 0.356 & $0.353 \mathrm{E}-\mathrm{F}$ \\
\hline 21 & 0.358 & 0.357 & 0.357 & $0.357 \mathrm{E}-\mathrm{F}$ \\
\hline 24 & 0.378 & 0.415 & 0.420 & $0.404 \mathrm{DE}$ \\
\hline 27 & 0.537 & 0.435 & 0.369 & $0.447 \mathrm{C}-\mathrm{E}$ \\
\hline 44 & 0.471 & 0.583 & 0.595 & $0.550 \mathrm{~A}-\mathrm{D}$ \\
\hline 79 & 0.212 & 0.974 & 0.374 & $0.520 \mathrm{~B}-\mathrm{D}$ \\
\hline 81 & 0.508 & 0.353 & 0.563 & $0.475 \mathrm{~B}-\mathrm{E}$ \\
\hline 91 & 0.742 & 0.718 & 0.647 & $0.703 \mathrm{~A}$ \\
\hline 96 & 0.468 & 0.532 & 0.471 & $0.490 \mathrm{~B}-\mathrm{E}$ \\
\hline 98 & 0.553 & 0.600 & 0.538 & $0.564 \mathrm{~A}-\mathrm{C}$ \\
\hline 99 & 0.418 & 0.424 & 0.429 & $0.424 \mathrm{C}-\mathrm{E}$ \\
\hline Mean & 0.405 & 0.460 & 0.425 & 0.430 \\
\hline
\end{tabular}

\subsection{Root length (cm)}

Genotype (dF:16; Ms:9.212), $\mathrm{NaCl}(\mathrm{dF}: 2 ; \mathrm{Ms}: 148.773)$ and genotype $\times \mathrm{NaCl}(\mathrm{dF}: 32 ; \mathrm{Ms}: 2.948)$ interaction had statistically different $(p<0.01)$. In three different $\mathrm{NaCl}$ doses, maximum root length was reached without any $\mathrm{NaCl}$ application $\left(0 \mathrm{mmhos} \mathrm{cm}^{-1}\right)(4.412 \mathrm{~cm})$, while minimum root length was obtained in $8 \mathrm{mmhos} \mathrm{cm}^{-1} \mathrm{NaCl}$ dose $(1.004$ $\mathrm{cm}$ ). According to different $\mathrm{NaCl}$ doses, they could be numercally ranked as 0 mmhos cm $\mathrm{m}^{-1}>4 \mathrm{mmhos} \mathrm{cm}^{-1}>8 \mathrm{mmhos}$ $\mathrm{cm}^{-1}$ ) in the same order. "No.81" had the highest root length $(4.163 \mathrm{~cm})$ as compared to other genotypes. However, significant reductions in root growth were observed under salt stress. The shortest root length was determined in "No.12" $(1.127 \mathrm{~cm})$. As it is mentioned about genotype $\times \mathrm{NaCl}$ applications, "No.81" was the superior genotype $(7.827 \mathrm{~cm})$ without any $\mathrm{NaCl}$ application. While "No.44" and $8 \mathrm{mmhos} \mathrm{cm}^{-1} \mathrm{NaCl}$ application was the worst $(0.510 \mathrm{~cm})$. "No.20" and "No.24" genotypes made the highest root growth $(1.557 \mathrm{~cm}$ and $1.563 \mathrm{~cm}$ respectively) and in the same Duncan

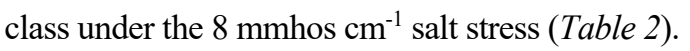

In this study, increased $\mathrm{NaCl}$ doses led to a decrease in root length (Figure 1). Without any $\mathrm{NaCl}$ application to 8 mmhos $\mathrm{cm}^{-1} \mathrm{NaCl}$ dose, it was observed approximately $75 \%$ loses of root length. $\mathrm{NaCl}$ stress caused a drastically effect on the roots as compared to shoots (Saha et al., 2010). Also, the roots absorb water and nutrients from soil. Thus, its length provides very important tip to the response of plants to salinity stress (Kaya and Ozturk, 2003; Moose and Mumm, 2008; Mohammed and Majid, 2013). Therefore, progressive decreases in seed germination, plant height, shoot and root length, dry matter, biomass, root, stem and leaf weights were observed with increased salinity stress of mung bean (Bhattacharjee et al., 2000; Raptan et al., 2001; Misra and Dwivedi, 2004; Rabie, 2005). Similar studies have been reported about the effects of root and shoot length under salinity stress conditions (Misra et al., 1996; Promila and Kumar, 2000; Mohammed, 2007; Cakmakci and Dallar, 2019).

\subsection{Shoot length $(\mathrm{cm})$}

According to varience analysis, genotype (dF:16; Ms:4.429), $\mathrm{NaCl}$ (dF:2; Ms:55.365) and genotype $\times \mathrm{NaCl}$ interaction (dF:32; Ms:1.267) had statistically different for the shoot length $(p<0.01)$. Shoot length values ranged 0.573$5.413 \mathrm{~cm}$. Also, maximum shoot length was observed in "No. 20" $(5.413 \mathrm{~cm})$ without any application of $\mathrm{NaCl}(0$ mmhos $\left.\mathrm{cm}^{-1}\right)$. And, minimum shoot length was determined in "No.99" $(0.573 \mathrm{~cm})$. Based on application of different $\mathrm{NaCl}$ doses, they could be numercally ranked as 0 mmhos $\mathrm{cm}^{-1}>4 \mathrm{mmhos} \mathrm{cm}^{-1}>8 \mathrm{mmhos} \mathrm{cm}^{-1}$ ) in descending order. Besides, it is ranged among 1.312-3.346 cm. In seventeen genotypes of mung bean, "No.20 "had the longest shoot length $(3.116 \mathrm{~cm})$, while "No.12 "had the smallest $(0.927 \mathrm{~cm})$. In addition, genotype "No.20" was the most developed shoots $(2.033 \mathrm{~cm})$ under the highest salt stress (Table 2). 
In the lights of these results; the shoot length was influenced by different genotypes and $\mathrm{NaCl}$ doses in the study (Figure 1). There is adversible effect between $\mathrm{NaCl}$ doses to shoot length. Misra et al. (1996) and Promila and Kumar (2000) stated that salt stress is reported to cause decline in shoot lengths, which was parallel to this study. When $\mathrm{NaCl}$ doses or salinity stress increased, shoot length decreased (Maliwal and Paliwal, 1982; Hug and Larher, 1983; Misra et al., 1996; Misra and Dwivedi, 2004). Most of mung bean genotypes can tolarate salt to extent to 9-18 m mhos cm${ }^{-1}$ (7.2-14.4 mmhos $\mathrm{cm}^{-1}$ ) (Singh and Singh, 2011). Also, Paliwal and Maliwal (1980) reported that mungbean seeds could tolerate 6 mmhos $\mathrm{cm}^{-1}\left(4.8 \mathrm{mmhos} \mathrm{cm}^{-1}\right)$ salinity. If the shoot length behaviour of seventeen genotypes and $\mathrm{NaCl}$ doses were compared, "No.20" and 0 mmhos $\mathrm{cm}^{-1} \mathrm{NaCl}$ dose were most favorable genotype and dose in this study.

\subsection{Fresh weight (g)}

Genotype (dF:16; Ms:1.382) and $\mathrm{NaCl}$ doses (dF:2; Ms:4.718) had statistically different ( $p<0.01)$, while genotype $\times \mathrm{NaCl}$ doses interaction had non significant effect. Fresh weight values ranged among 0.677-2.829 g. Maximum fresh weight was determined in without any application of $\mathrm{NaCl}$ doses $\left(0 \mathrm{mmhos} \mathrm{cm}^{-1}\right)(2.829 \mathrm{~g})$. Minimum fresh weight was noted in $8 \mathrm{mmhos} \mathrm{cm}^{-1} \mathrm{NaCl}$ dose $(0.677 \mathrm{~g})$. They could be numerically ranked as 0 mmhos $\mathrm{cm}^{-1}>4$ mmhos cm$\left.{ }^{1}>8 \mathrm{mmhos} \mathrm{cm}^{-1}\right)$ in the same order. As we talk about genotypes, "No.96" had the highest fresh weight $(2.511 \mathrm{~g})$, while "No.5" was the lowest one (1.041 g) (Table 2.).

Fresh weight was effected by genotypes and $\mathrm{NaCl}$ doses like shoot length, as we mentioned up there (Figure 1). If $\mathrm{NaCl}$ doses increased, it was found that fresh weight was decreased, except "No.20" (Table 2). It is seen that increasing $\mathrm{NaCl}$ doses affected on fresh weight negatively like root and shoot length. Ashraf and Rasul (1988), Misra et al. (1996), Misra and Dwivedi (2004) mentioned in their study that fresh weight of root and shoot length decreased with increased salinity, which was supported to this study. In their study, Misra and Dwivedi (2004) lose approximately $80.00 \%$ of fresh weight and $72.50 \%$ dry weight with increasing $\mathrm{NaCl}$ doses as compared to their control values. In this study, this loses reached $30 \%$, as maximum.

\subsection{Dry weight (g)}

Genotype (dF:16; Ms:0.195) was found significantly different ( $p<0.05)$. $\mathrm{NaCl}(\mathrm{dF}: 2 ; \mathrm{Ms}: 0.039)$ and genotype $\times$ $\mathrm{NaCl}$ doses had non significant effect for dry weight. Dry weight values ranged $0.187-0.974 \mathrm{~g}$. Application of $4 \mathrm{mmhos}$ $\mathrm{cm}^{-1} \mathrm{NaCl}$ dose was the most heaviest application $(0.974 \mathrm{~g})$. Without any application of $\mathrm{NaCL}(0 \mathrm{mmhos} \mathrm{cm}-1)$ was the lighest $(0.187 \mathrm{~g})$. And, they are numerically sorted by $4 \mathrm{mmhos} \mathrm{cm}^{-1}>8 \mathrm{mmhos} \mathrm{cm}^{-1}>0 \mathrm{mmhos} \mathrm{cm}^{-1}$, respectively. It is understand that 4 mmhos $\mathrm{cm}^{-1} \mathrm{NaCl}$ dose was the superior one as compared to other doses. Acccording to the different genotypes, "No. 91 " was the most heaviest genotype $(0.703 \mathrm{~g})$, while "No. 5 " was the lightest genotype $(0.214$ g).

On the contrary of the fresh weight, dry weight increased with increased $\mathrm{NaCl}$ doses. This might be linked due to decreased mobilization of reserve food materials from cotyledons at higher salinity levels (as shoot contains cotyledons) (Misra and Dwivedi, 2004). There are similar suggestions and studies about this knowledge (Sheroan and Garg, 1978; Dubey, 1985). 

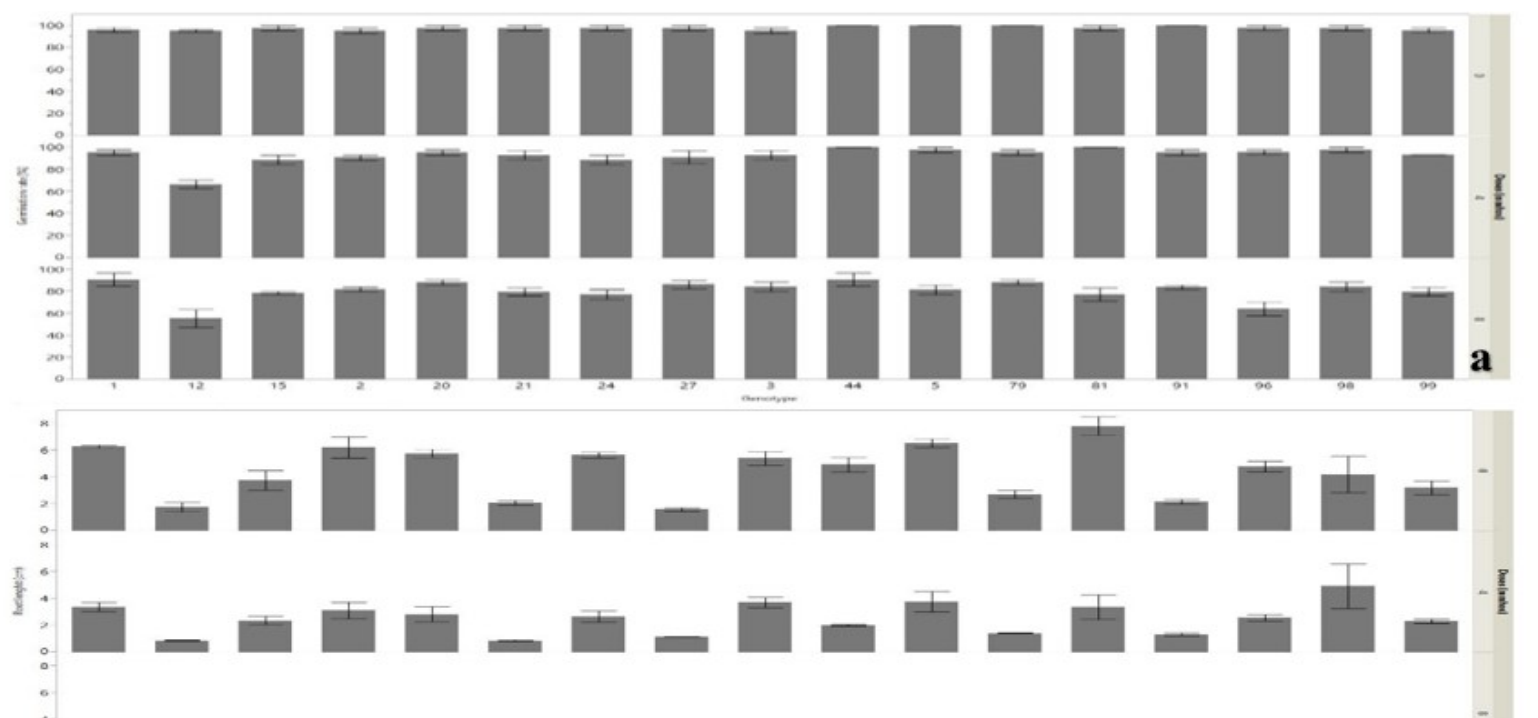

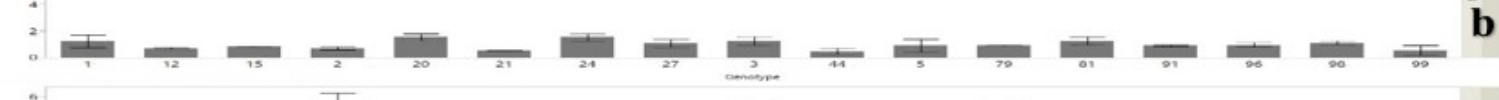

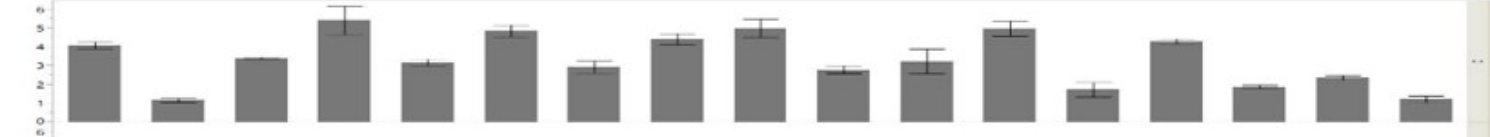

咅: :士
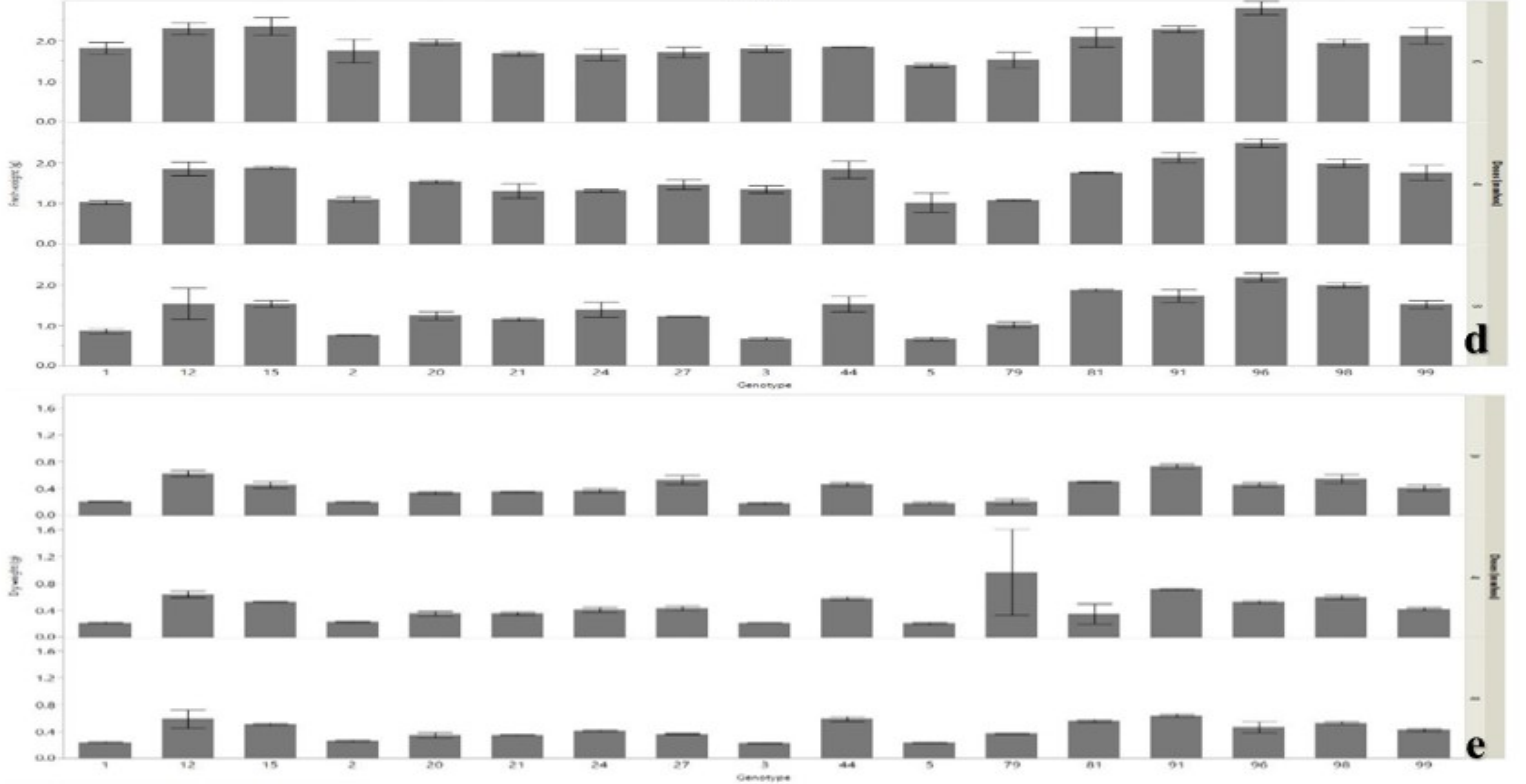

Figure 1. The effects of different salinity levels on mung bean genotypes (a: germination rate, b: root length, c: shoot length, d: fresh weight, e: dry weight) 


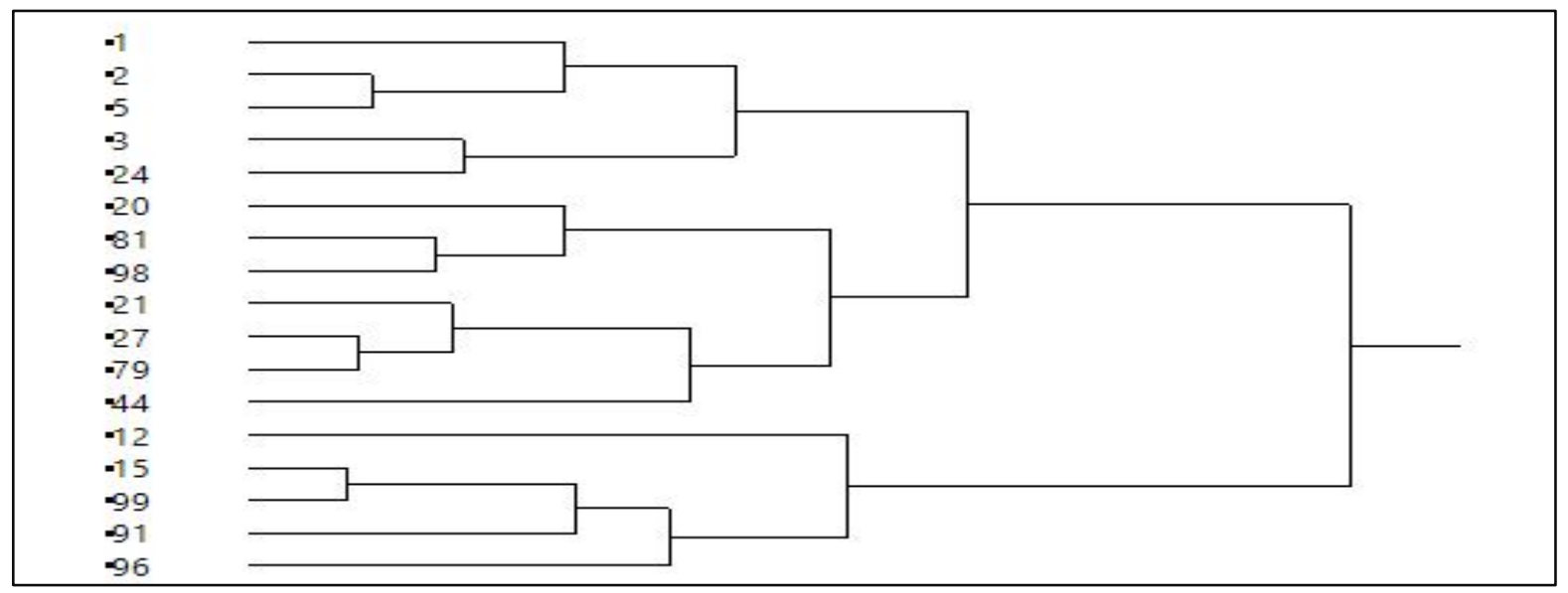

Figure 2. Dendrogram based on salt tolerance parameters of germination period of Mung bean genotypes

The dendogram showing the proximity-distance degrees of mung bean genotypes in terms of the investigated properties is given in Figure 2. According to the results, genotypes were divided into 4 main clusters. "No 15" and "No 99" genotypes were the closest to each other in terms of the characteristics studied. "No 1" and "No 12" were the most different genotypes in terms of these characteristics.

\section{Conclusions}

Based on the data obtained from the studies, "No.20" and "No.24" genotypes were found to be more tolerant to salt stress than the other genotypes used in the experiments. Consequently, it is suggested that it can be used as parental plants in the breeding of new varieties tolerant to salinity in the future. 


\section{References}

Abd-Alla, M.H., Vuong, T.D. and Harper, J.E. 1998. Genotypic differences in nitrogen fixation response to NaCl stress in intact and grafted soybean. Crop Science 38: 72.

Ashraf, M. and Rasul, E. 1988. Salt tolerance of mung bean (Vigna radiata (L.) Wilczek) at two growth stages. Plant and Soil, $110(1), 63-67$.

Ashraf, Muhammad, and Majid, R. Foolad. 2013. "Crop breeding for salt tolerance in the era of molecular markers and marker-assisted selection." Plant Breeding 132.1: 10-20.

Bhattacharjee, A., Maity, S., Banerjee, G., Roy, M., Pal, C., Pal, B. and Chakrabarti, D. 2000. Chemical induced prolongation of seed viability and stress tolerance capacity of mung bean seedlings. Seed Science and Technology (Switzerland), 28(1), 155-162.

Cakmakci, S. and Dallar, A. 2019. Farklı Sıcaklık ve Tuz Konsantrasyonlarının Bazı Silajlık Mısır Çeşitlerinin Çimlenme Özellikleri Üzerine Etkileri. Tekirdağ Ziraat Fakültesi Dergisi, 16(2), 121-132.

Culha, S. and Cakirlar, H. 2011. The Effect of Salinity on Plants and Salt Tolerance Mechanisms. Afyon Kocatepe University Journal of Sciences and Engineering, Vol: 11(2):11-34.

Demirbas, S. and Balkan, A. 2018. Tuz Stresi Koşullarında Bazı Tritikale Çeşitlerinin Hidrojen Peroksit Ön Uygulamasına Tepkileri. Tekirdağ Ziraat Fakültesi Dergisi, 15(2), 5-13.

Dubey, R.S. 1985. Effect of salinity on nucleic acid metabolism of germinating rice seeds differing in salt tolerance, Plant Physiol. Biochemistry. Vol:12, p:9-16.

HanumanthaRao, B., Nair, R. M. and Nayyar, H. 2016. Salinity and high temperature tolerance in mungbean [Vigna radiata (L.) Wilczek] from a physiological perspective. Frontiers in Plant Science, 7, 957.

Hug, S.M.I., Larher, F. 1983. Osmoregulation in higher plants: effects of NaCI salinity on non-nodulated Phaseolus aureus L. I. Growth and mineral content. New phytologist. 93: 203-208.

ISTA, 2016. International Seed Testing Association. International Rules for Seed Testing.

Kaya, M. D., Ipek, A. and Ozturk, A. 2003. Effects of different soil salinity levels on germination and seedling growth of safflower (Carthamus tinctorius L.). Turkish Journal of Agriculture and Forestry, 27(4), 221-227.

Maliwal, G.L. and Paliwal, K.V. 1982. Salt tolerance of some mungbean (Vigna radiata), urdbean (Vigna mungo) and gaur (Cyamopsis tetragonoloba) varieties at germination and early stages. Legume Research 5: 23-30.

Misra, N., Murmu, B., Singh, P. and Misra, M. 1996. Growth and proline accumulation in mungebean seedlings as affected by sodium chloride. Biologia Plantarum 58: 531-536.

Misra, N. and Dwivedi, U. N. 2004. Genotypic difference in salinity tolerance of green gram cultivars. Plant Science, 166(5), 1135-1142.

Mohammed, A. H. M. A. 2007. Physiological aspects of mungbean plant (Vigna radiata L. Wilczek) in response to salt stress and gibberellic acid treatment. Research Journal of Agriculture and Biological Sciences, 3, 200-213.

Moose, S. P. and Mumm, R. H. 2008. Molecular plant breeding as the foundation for 21st century crop improvement. Plant physiology, 147(3), 969977.

Ozgen, M., Ertunc, F., Kinaci, G., Yildiz, M., Birsin, M., Ulukan, H., Koyuncu, N. and Sancak, C. 2005. New approaches and applications in agricultural Technologies. Turkey Agricultural Engineering Technical Conference 3-7 January 2005 p.

Paliwal, K. V. and Maliwal, G. L. 1980. Growth and nutrient uptake relationship of some crops in saline substrate. Annals of the Arid Zone 19: 251253.

Parida, A.K. and Das, A.B. 2005.Salt tolerance and salinity effects on plants: a review. Ecotoxicology and Environmental Safety. 60, 324-349.

Pitman, M.G. and Lauchli, A. 2002. Global Impact of Salinity and Agricultural Ecosystems. Salinity: Environment-Plants-Molecules Published by Kluwer Academic Publishers. Dordrecht. The Netherlands. 522 p.

Promila, K. and Kumar, S. 2000. Vigna radiata seed germination under salinity. Biologia Plantarum 43: 423-426.

Rabie, G. H. 2005. Influence of arbuscular mycorrhizal fungi and kinetin on the response of mungbean plants to irrigation with seawater. Mycorrhiza, $15(3), 225-230$

Raptan, P. K., Hamid, A., Khaliq, Q. A., Solaiman, A. R. M., Ahmed, J. U. and Karim, M. A. 2001. Salinity Tolerance of Blackgram and Mungbean. 한국작물학회지, 46(5), 380-386.

Saha, P., Chatterjee, P. and Biswas, A.K. 2010. NaCl pretreatment alleviates salt stress by enhancement of antioxidant defense and osmolyte accumulation in mungbean (Vigna radiata L. Wilczek). Indian Journal of Experimental Biology 48: 593-600.

SAS, Institute Inc. 2015. JMP® Statistical Discovery Software, version 12.0; SAS Institute Inc., Cary, NC, USA, 2015.

Sheoran, I.S. and Garg, O.P. 1978. Effect of salinity on activities of RNAse, DNAse and protease during germination and early seedling growth of mung bean, Physiologia Plantarum, Vol: 44, p:171-174. 
Siemonsma, J.S. and Na Lampang, A. 1992. Vigna radiata (L.) Wilczek. In: Plant Resources of South-East Asia 1, Pulses, Editors: van der Maesen L.J.G. and Somaatmadja S., Pudoc, Leiden, Netherlands, pp. 71-74.

Singh, D. P. and Singh, B. B. 2011. Breeding for tolerance to abiotic stresses in mungbean. Food Legumes, 24(2), 83-90.

Tuteja, N. 2007. Mechanisms of High Salinity Tolerance in Plants. Methods in Enzymology, 428: 419-438.

Yıldız, M. and Ozgen, M. 2004. The effect of a submersion pretreatment on in vitro explant growth and shoot regeneration from hypocotyls of flax (Linum usitatissimum). Plant Cell, Tissue and Organ Culture, 77(1):111-115.

Zhu J.K. 2002. Salt and Drought Stress Signal Transduction in Plants. Annual Review of Plant Biology. 53:247-273. 